\title{
Minimization of risks in pension funding by means of contributions and portfolio selection ${ }^{\text {th }}$
}

\author{
Ricardo Josa-Fombellida ${ }^{1}$, Juan Pablo Rincón-Zapatero*,2 \\ Dpto. de Economía Aplicada (Matemáticas), Universidad de Valladolid, Avda. Valle Esgueva 6, 47011 Valladolid, Spain
}

\begin{abstract}
We consider a dynamic model of pension funding in a defined benefit plan of an employment system. The prior objective of the sponsor of the pension plan is the determination of the contribution rate amortizing the unfunded actuarial liability, in order to minimize the contribution rate risk and the solvency risk. To this end, the promoter invest in a portfolio with $n$ risky assets and a risk-free security. The aim of this paper is to determine the optimal funding behavior in this dynamic, stochastic framework.
\end{abstract}

JEL classification: G23; G11

Subj. Class.: E13; B81

Keywords: Pension funding; Contribution rate risk; Solvency risk; Asset allocation; Stochastic control

\section{Introduction}

In a defined benefit plan of an employment system, it is common to apply the so known Actuarial Cost Methods, which allow us to determine an ideal contribution rate or normal cost and an ideal fund level (that could be related with the actuarial liability) in such a way that the benefits promised to a collective of members be guaranteed along the time. Actually, the existence of unexpected disturbances can make the evolution of the plan will not be in accordance with the valuation designed at the beginning. Therefore, the contribution rate must be the normal cost plus a positive or negative increment, called the supplementary cost. Of course, the promoter of the fund must planning how to drive the unfunded actuarial liability to zero. We suppose that the plan is built with the contributions and investment earnings. The sponsoring employer controls the contribution rate and the amount invested in a portfolio composed of $n$ risky assets and a risk-free security. No shortselling is allowed but the manager have the possibility of borrowing.

\footnotetext{
We are grateful to the anonymous referee who carefully read the manuscript and suggested a number of improvements ${ }^{*}$ Corresponding author

E-mail address: zapatero@eco uva es (J P Rincón-Zapatero)

${ }^{1}$ The research of this author was supported by Investigation Projects V12/99 and VA108/01 of Consejería de Educación y Cultura de la Junta de Castilla y León, Spain

2 The research of this author was supported by Investigation Project PB98-0393 of Dirección General de Enseñanza Superior e Investigación Científica and VA108/01 of Consejería de Educación y Cultura de la Junta de Castilla y León, Spain
} 
In Haberman (1993), Haberman and Sung (1994), Haberman (1997), two main types of risks are considered which the pension plan is confronted, the contribution rate risk and the solvency risk. The first is measured with the size of the deviations of the contributions from the normal cost and it is related with the stability of the plan. The second risk is measured with the size of the unfunding actuarial liability and it can serve as an indicator of the security of the plan.

The promoter wishes to control the stability and security of the pension plan by minimizing some convex combination of both types of risk. In this way, the weight in the convex combination measures the relative importance of the risk for the promoter. The attained solution is a Pareto optimum of this multi-objective problem. This means that there is no possible reduction of one of the risks without augmenting the other one.

Another important feature of the model is the presence of an unbounded horizon. We suppose that there is not a finish time for the plan but it extends forever and that the preferences of the sponsor are more concerned with the short run that with the long run; this is modeled by introducing a positive discount factor in the objective functional.

The assets of the fund can be invested in $n+1$ securities, $n$ of them following correlated geometrical Brownian motions. The risk-free security is constant in time. It then follows that the assets of the fund obeys a stochastic differential equation closely related with that proposed in Merton (1971) for portfolio and consumption selection.

Haberman and Sung (1994) considers the minimization of a linear combination of the aforementioned risks on a finite horizon both in deterministic and stochastic frameworks. The authors do not contemplate the investment as an instrumental variable but all the assets of the fund are invested at a random rate of return. As a consequence the optimal control problem studied in Haberman and Sung (1994) turns out to be one of the linear-quadratic type, that can be explicitly solved. Although in the model we propose the objective functional is quadratic, the dynamics is nonlinear, so the control problem is not linear-quadratic. However, we are able to find a closed form solution for the problem of the optimal management of pension funding.

O'Brien (1987) analyze a stochastic optimal control problem which shows two sources of uncertainty, the investment returns and the benefit outcome. The author makes a linear approximation of the exponential fund model, see Bowers et al. $(1976,1979)$, to retain analytical tractability of the problem. However, no investment decisions are available for the manager, who wishes to maintain a constant fund ratio (with respect to the actuarial liability), and penalizes fluctuations of the contribution rate from zero.

Boulier et al. (1995) study a related model of pension fund management. They consider the case that pension payments grow at a fixed, deterministic rate. The manager try to minimize the total amount of contributions discounted with a positive parameter. They allow the contributions be invested in a portfolio composed of two financial assets. The authors find that there is a threshold value for the fund such that the optimum contribution and inversion rates are linear with respect to the fund size at the left of threshold, and zero at the right.

In a subsequent paper, Boulier et al. (1996) adds an upper bound on the maximum contribution rate. In this case the asset allocation strategies are no longer linear and there is no a closed solution.

Cairns $(1995,1996)$ obtains similar results for a more general problem where the loss function is of the same form suggested by Haberman and Sung (1994). This fact makes the optimal control variables differ somewhat of those founded by Boulier et al. (1995). In the papers by Cairns it turn out to be linear functions.

Cairns (2000) provides a more general framework for the modeling and control of pension funds. He considers $n$ risky assets and a risk-free asset as well as randomness in the level of benefit outgo. There is no explicit reference to the actuarial liability nor to the normal cost rate. Besides a quadratic loss function, power and exponential objective functions are investigated. Furthermore, constraints on the contribution and asset-allocation strategies are allowed. However, there is no proof of their optimality in the constrained case.

Our main objective in this paper is to find the optimal contribution and investment rates and to give a rigorous proof of their optimality in the presence of shortselling constraints. Selling short implies a somewhat speculative behavior that seems to be not adequate for the management of the comprised liabilities. As will be shown in the paper, this restriction motivates that the supplementary cost is piecewise linear with respect to the unfunded actuarial liability. The constant of proportionality is higher for values of the fund above the actuarial liability. This fact seems to be omitted in previous works on the subject. We also find that no inversion takes place in the risky assets whenever 
the fund is above the actuarial liability and that borrowing at the risk-free rate to invert in the risky assets is optimal for values of the fund below a fixed value.

The paper is structured as follows. In Section 2, we setup the model as a controlled diffusion problem. In Section 3 , we outline the dynamic programming approach and we find the optimal controls in feedback form, obtaining, roughly speaking, that the portfolio choice and contribution rate are (piecewise) proportional to the unfunded actuarial liability. Section 4 is devoted to establish some conclusions. Finally, in Appendix A are proved some stability and convergence properties of the fund.

\section{Mathematical model}

All the variables listed is this section are related to all the participants in the aggregate pension fund we will consider. We assume that the actuarial valuation to estimate the main components of the plan are done at each instant of time.

We denote $F(t)$ as the value of the assets forming the fund at time $t ; C(t)$ is the contribution rate made by the sponsor in order to accrue the amount of the defined benefit at the moment of retirement; the defined benefits are denoted by $P$; the normal cost for all participants, by NC; the actuarial liability, by AL; the unfunded actuarial liability, by UAL (this is simply the difference between $\mathrm{AL}$ and $F(t)$ ) and the supplementary contribution rate amortizing UAL at time t, by SC (this is simply the difference between $C(t)$ and $\mathrm{NC}$ ).

Let us observe that we have considered constant values of $P, \mathrm{NC}$ and $\mathrm{AL}$. This is justified, as in Haberman and Sung (1994), if the population in the pension plan is stationary from the start and there is no salary increase (or there is a fixed rate of salary inflation; in this case the rate of return is net the salary inflation).

If we assume that the valuation of the plan is done with a constant rate $\delta$, then the main components of the plan are linked by the equation

$$
\delta \mathrm{AL}+\mathrm{NC}-P=0,
$$

as is shown in Bowers et al. (1976).

The sponsoring employer manages the funding process by making a portfolio choice of $n$ risky assets $S^{1}(t), \ldots, S^{n}(t)$ and a bond $S^{0}(t), 0 \leq t<\infty$, with dynamics given by the equations

$$
\begin{aligned}
& \mathrm{d} S^{0}(t)=r S^{0}(t) \mathrm{d} t, \quad S^{0}(0)=1, \\
& \mathrm{~d} S^{i}(t)=S^{i}(t)\left(b_{i} \mathrm{~d} t+\sum_{j=1}^{n} \sigma_{i j} \mathrm{~d} W_{j}(t)\right), \quad S^{i}(0)=s_{i}, \quad 1 \leq i \leq n .
\end{aligned}
$$

Here $b_{i}$ and $\sigma_{i j}, 1 \leq i, j \leq n$ are positive constants. The vector $\mathbf{W}(t)=\left(W_{1}(t), \ldots, W_{n}(t)\right)^{\mathrm{T}}$ is an $n$-dimensional standard Brownian motion defined on a probability space $(\Omega, \mathcal{F}, P)$, where $\left\{\mathcal{F}_{t}\right\}$ denotes the completion of the filtration $\sigma\{\mathbf{W}(s) \mid 0 \leq s \leq t\}$.

We have supposed that the liabilities are known deterministically. In the case that the liability cash flows were uncertain, it would be possible to use a bond with cash flows that matched the liabilities. This could be done, e.g., through the calculation of mean terms of the values of the assets of the fund and the values of the liabilities, as in the theory of matching, see Daykin et al. (1994). The concept of matching implies a balance between the value of the fund and the liabilities that is preserved against changes in interest rates.

We suppose that the interest rate $r$ is strictly smaller than the mean rates of return $b_{i}, 1 \leq i \leq n$. Next we introduce the matrix $\sigma=\left(\sigma_{i j}\right)$ and the column vectors $\mathbf{b}=\left(b_{1}, \ldots, b_{n}\right)^{\mathrm{T}}, \boldsymbol{\Lambda}(t)=\left(\lambda_{1}(t), \ldots, \lambda_{n}(t)\right)^{\mathrm{T}}$.

A portfolio process or trading strategy $\Lambda(t)$ is an $\mathbb{R}^{n}$-measurable process adapted to $\left\{\mathcal{F}_{t}\right\}$ such that

$$
\int_{0}^{\infty}\|\boldsymbol{\Lambda}(s)\|^{2} \mathrm{~d} s<\infty \text { a.s., for every } t<\infty .
$$


Here $\lambda_{i}(t) \geq 0$ denotes the proportion of the fund assets invested by the promoter in asset $i, 0 \leq i \leq n$. As explained in Section 1, the non-negativity constraint on $\lambda_{i}$ avoids shortselling.

The contribution rate process $C(t)$ is a measurable adapted process with respect to $\left\{\mathcal{F}_{t}\right\}$ verifying

$$
\int_{0}^{t}|C(s)|^{2} \mathrm{~d} s<\infty \text { a.s., for every } t<\infty .
$$

and

$$
E_{F_{0}} \int_{0}^{\infty} \exp (-\rho t)\left(\beta \mathrm{SC}^{2}(t)+(1-\beta) \mathrm{UAL}^{2}(t)\right) \mathrm{d} t<\infty .
$$

Here, $E_{F_{0}}$ denotes conditional expectation by $F_{0}$. The class of admissible controls is denoted by $\mathcal{A}_{F_{0}}$.

The quantity $\lambda_{i}(t) F(t)$ represents the total amount invested in asset $i, 1 \leq i \leq n$, hence $\left(1-\sum_{i=1}^{n} \lambda_{i}(t)\right) F(t)$ is the amount invested in the bond. The total contribution made up to time $t$ is given by $\int_{0}^{t} C(s) \mathrm{d} s$. Along the lines of Merton (1971), we suppose that changes in the fund level derive only from changes in the asset prices, interest of the bond and from the contribution. In consequence:

$$
\mathrm{d} F(t)=F(t)\left(\sum_{i=1}^{n} \lambda_{i}(t) \frac{\mathrm{d} S^{i}(t)}{S^{i}(t)}\right)+F(t)\left(1-\sum_{i=1}^{n} \lambda_{i}(t)\right) \frac{\mathrm{d} S^{0}(t)}{S^{0}(t)}+(C(t)-P) \mathrm{d} t .
$$

Taking into account (2)-(4) we obtain that the fund amount satisfies the following stochastic differential equation:

$$
\mathrm{d} F(t)=\left(r F(t)+\sum_{i=1}^{n} \lambda_{i}(t)\left(b_{i}-r\right) F(t)+C(t)-P\right) \mathrm{d} t+\sum_{i=1}^{n} \sum_{j=1}^{n} \lambda_{i}(t) \sigma_{i j} F(t) \mathrm{d} W_{j}(t)
$$

with initial condition $F(0)=F_{0}>0$.

The symmetric matrix $\Sigma=\sigma \sigma^{\mathrm{T}}$ is positive definite. We denote $\theta=\sigma^{-1}(\mathbf{b}-r \mathbf{1})$ the market price of risk, where 1 denotes a (column) vector of $1 \mathrm{~s}$. Obviously, $\theta^{\mathrm{T}} \theta$ is a positive scalar.

Now, we turn with the preferences of the controller. We assume that he or she wish to minimize a convex combination of the contribution rate risk and the solvency risk. In consequence, the objective functional to be minimized over $\mathcal{A}_{F_{0}}$ is

$$
J(F, C, \boldsymbol{\Lambda})=E_{F_{0}} \int_{0}^{\infty} \exp (-\rho t)\left(\beta \mathrm{SC}^{2}(t)+(1-\beta) \mathrm{UAL}^{2}(t)\right) \mathrm{d} t .
$$

The parameter $\beta$ verifies $0<\beta \leq 1$ and is a weighting factor reflecting the relative importance of one type of risk with respect to the other one. The restriction in the range of possible values of $\beta$ means that the sponsor choose a compromise solution or Pareto optimal solution in the multi-objective problem arising in this decision model. There is also a positive actualization rate, $\rho$. A high actualization rate implies that the promoter is more concerned with the present than with the distant future.

Throughout the paper we make the assumption that $\delta$ equals $r$, that is to say, the valuation force of interest coincides with the rate of return of the bond. Having into account that there is no uncertainty in the liability cash flows, we are legitimated to discount them at a risk-free rate of interest. This would not be appropriate if there was some random element in the benefits. As will be shown below, this hypothesis implies that full investment in the risk-free bond is optimal when the assets of the fund are above the prescribed target. This behavior of the optimal solution is similar to the one reported in Boulier et al. (1995). Let us note that in this paper there is no a priori ideal level for the fund, but this appears as the discounted value of all future cash flows due to pension payment in an infinite horizon. 


\section{Optimal feedback pension funding control}

In this section, we establish some properties of the value function of the control problem introduced in Section 2 and we prove that it is a generalized solution to the Hamilton-Jacobi-Bellman equation (HJB henceforth). The value function is defined as

$$
\hat{V}(F)=\inf _{(C, \boldsymbol{\Lambda}) \in \mathcal{A}_{F_{0}}}\{J(F, C, \boldsymbol{\Lambda}) \mid \text { s.t. }(5)\} .
$$

The function $\hat{V}(F)$ is the minimum value of the deviations from the objectives when the initial wealth in the fund is $F$. Given that the problem is autonomous and the horizon is unbounded, we may suppose that $\hat{V}$ is time-independent. It is clear that the value function so defined is non-negative, strictly convex and that $\hat{V}(\mathrm{AL})=0$.

As is well known since Bellman (1957), the knowledge of the value function implies the knowledge of the optimal controls (at least in an implicit way). The connection between value functions in optimal control theory (deterministic or stochastic) and optimal feedback controls is accomplished by the HJB equation, see Fleming and Soner (1992). For our problem of optimal pension funding, the HJB equation becomes:

$$
\begin{aligned}
& \rho V(F) \\
& \quad=\min _{C, \boldsymbol{\Lambda} \geq 0}\left\{\beta(C-\mathrm{NC})^{2}+(1-\beta)(\mathrm{AL}-F)^{2}+\left(r F+\boldsymbol{\Lambda}^{\mathrm{T}}(\mathbf{b}-r \mathbf{1}) F+C-P\right) V^{\prime}(F)+\frac{1}{2} \boldsymbol{\Lambda}^{\mathrm{T}} \Sigma \boldsymbol{\Lambda} F^{2} V^{\prime \prime}(F)\right\} .
\end{aligned}
$$

Whenever a solution $V$ of the above equation is smooth enough, the minimizing arguments are given by

$$
\begin{aligned}
& \tilde{C}\left(V^{\prime}(F)\right)=\mathrm{NC}-\frac{1}{2 \beta} V^{\prime}(F), \\
& \tilde{\mathbf{\Lambda}}\left(V^{\prime}(F), V^{\prime \prime}(F)\right)=\left(-\frac{V^{\prime}(F)}{F V^{\prime \prime}(F)} \Sigma^{-1}(\mathbf{b}-r \mathbf{1})\right)_{+} .
\end{aligned}
$$

Here, given $\mathbf{a}=\left(a_{1}, \ldots, a_{n}\right) \in \mathbb{R}^{n}, \mathbf{a}_{+}$denotes the vector which $i$-coordinate is $\max \left\{a_{i}, 0\right\}$. The equalities (7) and (8) are the link between the HJB equation and the optimal controls of the problem. Observe that the scalar magnitude in (8) is the inverse of the Arrow-Pratt measure of risk aversion of the value function.

In order to find $V$ satisfying (6) we make the following observations: (i) deviations of the wealth of the fund from the actuarial liability are penalized, so the investment in the risky assets must be zero if $F>\mathrm{AL}$, because their mean of return is higher than that of the bond, and (ii) the value function $\hat{V}$ is a solution of (6) if it is smooth enough. Since the running cost is quadratic we will postulate a piecewise quadratic value function of class $\mathcal{C}^{1}$ (although the dynamics is non-linear). ${ }^{3}$ The necessity for consider a piecewise smooth function comes from the impossibility of shortselling. Based on the above considerations, we make the guessing

$$
\tilde{\boldsymbol{\Lambda}}(F)= \begin{cases}-\frac{V^{\prime}(F)}{F V^{\prime \prime}(F)} \Sigma^{-1}(\mathbf{b}-r \mathbf{1}) & \text { if } F<\mathrm{AL}, \\ \mathbf{0} & \text { if } F>\mathrm{AL},\end{cases}
$$

where $\mathbf{0}$ is a (column) vector of $0 \mathrm{~s}$, and

$$
V(F)= \begin{cases}a(\mathrm{AL}-F)^{2} & \text { if } F<\mathrm{AL}, \\ \alpha(\mathrm{AL}-F)^{2} & \text { if } F>\mathrm{AL},\end{cases}
$$

\footnotetext{
${ }^{3}$ A function is of class $\mathcal{C}^{k}(\mathbb{R})$ if its first $k$-derivatives exists and are continuous functions on $\mathbb{R}$.
} 
as a smooth solution of (6) on the regions $F<\mathrm{AL}$ and $F>\mathrm{AL}$ with the exception of the point $F=\mathrm{AL}$. By substituting (10) in (6) we find that the positive constants $a, \alpha$, must satisfy the two equations

$$
\begin{aligned}
& a^{2}+\beta\left(\rho-2 r+\theta^{\mathrm{T}} \theta\right) a-\beta(1-\beta)=0, \\
& \alpha^{2}+\beta(\rho-2 r) \alpha-\beta(1-\beta)=0 .
\end{aligned}
$$

It is easy to see that (11) and (12) admits each one a unique positive solution verifying $a<\alpha$. It turns out that $V$ satisfies the conditions $V^{\prime}\left(\mathrm{AL}^{-}\right)=V^{\prime}\left(\mathrm{AL}^{+}\right)=0$ and $V^{\prime \prime}\left(\mathrm{AL}^{+}\right)=2 \alpha>2 a=V^{\prime \prime}\left(\mathrm{AL}^{-}\right)$.

We now proceed to confirm the validity of our conjecture, i.e., $V$ as defined in (10) coincides with the value function $\hat{V}$. To this end we will use a verification theorem based on a generalized Itô's rule.

Theorem 1. The optimum contribution rate in feedback form is given by

$$
C^{*}(F)= \begin{cases}\mathrm{NC}+\frac{a}{\beta}(\mathrm{AL}-F) & \text { if } F<\mathrm{AL}, \\ \mathrm{NC}+\frac{\alpha}{\beta}(\mathrm{AL}-F) & \text { if } F>\mathrm{AL} .\end{cases}
$$

The optimal investment policy is

$$
\boldsymbol{\Lambda}^{*}(F)= \begin{cases}\frac{\mathrm{AL}-F}{F} \Sigma^{-1}(\mathbf{b}-r \mathbf{1}) & \text { if } 0<F<\mathrm{AL}, \\ \mathbf{0} & \text { if } F>\mathrm{AL} .\end{cases}
$$

Here, $a$ is the unique positive solution of (11) verifying

$$
a>\beta\left(r-\theta^{\mathrm{T}} \theta\right),
$$

and $\alpha$ is the unique positive solution of (12) such that

$$
\alpha>\beta r .
$$

Proof. Denote $F^{*}$ the wealth of the fund associated with $C^{*}$ and $\Lambda^{*}$, when the initial condition is $F^{*}(0)=F$. The pair $\left(C^{*}, \Lambda^{*}\right)$ belongs to the admissible class of controls $\mathcal{A}_{F}$ because of Proposition 1 in Appendix $\mathrm{A}$, where it is proved that the expected values of $F^{*}$ and $C^{*}$ converge to $\mathrm{AL}$ and $\mathrm{NC}$, respectively.

Now we consider an arbitrary pair $(C, \boldsymbol{\Lambda}) \in \mathcal{A}_{F}$. Applying the generalized Itô's rule (Karatzas and Shreve, 1991, p. 219) to $\mathrm{e}^{-\rho t} V(t)$ we obtain, after taking expectations

$$
\begin{gathered}
\mathrm{e}^{-\rho t} E_{F} V(F(t))=V(F)+E_{F} \int_{0}^{t}\left[\frac{1}{2} \boldsymbol{\Lambda}^{\mathrm{T}}(s) \Sigma \boldsymbol{\Lambda}(s) F(s)^{2} V^{\prime \prime}(F(s))+\left(r F(s)+(\mathbf{b}-r \mathbf{1})^{\mathrm{T}} \boldsymbol{\Lambda}(s) F(s)\right.\right. \\
\left.+C(s)-P) V^{\prime}(F(s))-\rho V(F(s))\right] \mathrm{d} s .
\end{gathered}
$$

We have made use of the fact that $V^{\prime}$ is continuous, so there is no local time term in the stochastic integral. On the other hand, (6) implies

$$
\rho V(F) \leq \beta(C-\mathrm{NC})^{2}+(1-\beta)(\mathrm{AL}-F)^{2}+\left(r F+(\mathbf{b}-r \mathbf{1})^{\mathrm{T}} \boldsymbol{\Lambda} F+C-P\right) V^{\prime}(F)+\frac{1}{2} \boldsymbol{\Lambda}^{\mathrm{T}} \Sigma \boldsymbol{\Lambda} F^{2} V^{\prime \prime}(F)
$$

for all $F \neq \mathrm{AL}$, with equality whenever we replace $C$ by $C^{*}$ and $\Lambda$ by $\Lambda^{*}$. Integrating and taking expectations in (17) and making use of the existence of side limits of $V^{\prime \prime}$ in AL, we have 


$$
\mathrm{e}^{-\rho t} E_{F} V(F(t))+E_{F} \int_{0}^{t} \mathrm{e}^{-\rho s}\left[\beta(C(s)-\mathrm{NC})^{2}+(1-\beta)(\mathrm{AL}-F(s))^{2}\right] \mathrm{d} s \geq V(F)
$$

with equality when $C=C^{*}$ and $\Lambda=\Lambda^{*}$.

The transversality condition $\lim _{t \rightarrow \infty} \mathrm{e}^{-\rho t} E_{F} V(F(t))=0$ holds, because of Proposition 1 in Appendix A. Hence inequality (18) implies $J(F ; C, \boldsymbol{\Lambda}) \geq V(F)$ and $J\left(F ; C^{*}, \boldsymbol{\Lambda}^{*}\right)=V(F)$. It then follows that $\left(C^{*}, \boldsymbol{\Lambda}^{*}\right)$ is optimum on $\mathcal{A}_{F}$.

It is interesting to note that, as mentioned above, the value function is not of class $\mathcal{C}^{2}(\mathbb{R})$. This means that the smooth pasting conditions (see Krylov (1980, p. 32)), cannot be fulfilled by the value function of our problem, because $V^{\prime \prime}\left(\mathrm{AL}^{-}\right)=2 a<2 \alpha=V^{\prime \prime}\left(\mathrm{AL}^{+}\right)$.

Note that (6) is not uniformly elliptic because the second order term $\frac{1}{2} \Lambda^{\mathrm{T}} \Sigma \boldsymbol{\Lambda} F^{2} V^{\prime \prime}(F)$ becomes zero when $\Lambda=0$, so we cannot be sure of the existence of a smooth solution of the equation, see Krylov (1980). ${ }^{4}$ In fact, the optimal $\Lambda^{*}$ goes to zero as $F$ goes to AL. At this point it is worth comparing with the model of optimal consumption and portfolio choice with no shortselling, where the value function is proved to be of class $\mathcal{C}^{2}$, see Vila and Zariphopoulou (1997).

The expressions for the optimal rate of contribution and the optimal vector of investments given in the above theorem can be rephrased as

$$
\mathrm{SC}=\left\{\begin{array}{ll}
\frac{a}{\beta} \mathrm{UAL} & \text { if } \mathrm{UAL}>0, \\
\frac{\alpha}{\beta} \mathrm{UAL} & \text { if } \mathrm{UAL}<0,
\end{array} \quad \boldsymbol{\Lambda}^{*}(F) F= \begin{cases}\mathrm{UAL} \Sigma^{-1}(\mathbf{b}-r \mathbf{1}) & \text { if } \mathrm{UAL}>0, \\
\mathbf{0} & \text { if } \mathrm{UAL}<0 .\end{cases}\right.
$$

The total investment vector $\Lambda^{*} F$ is a constant proportion policy on the region UAL $>0$, because regardless the gap between the wealth of the fund and the goal, the proportion of wealth invested in the risky stocks is fixed. Thus the manager of the plan gets increasingly more cautious as the wealth of the fund takes closer values to the actuarial liability. This implies to invest less at each time, eventually reaching zero investment in the limit. Similar comments apply to the optimal contribution rate on each of the regions UAL $>0$ and UAL $<0$. Let us observe that the supplementary cost depends on $\beta$ but the investment strategy does not.

The optimal funding process can be summarized in the two following rules: (i) keep the supplementary cost proportional to the unfunded actuarial liability, with different constants of proportionality $a / \beta$ or $\alpha / \beta$, as soon as the wealth of the fund is below or above the actuarial liability, respectively and (ii) make an investment in the risky assets proportional to the unfunded actuarial liability whenever the wealth of the fund is below the objective. Do not invest anything in other case.

Although the investment behavior seems to be paradoxical, we must remember that the prior objective of the employer sponsor is to reduce the inherent risks of the process funding, and not to maximize the wealth of the fund.

It is very interesting to note that the optimal supplementary cost for this problem corresponds to a spread method of contribution, see Bowers et al. (1976), that is to say, it is proportional to the unfunded actuarial liability. This funding method is very used in the literature and has been proved to have good properties for the stabilization of the pension plan. Here we find another justification for this method, because it arise naturally as a consequence of an optimal or extremal aiming of the controller. Haberman and Sung (1994) and Owadally and Haberman (1999) lead to similar results in a discrete time formulation.

Let us observe that Theorem 1 shows that the constant of proportionality is different in the regions $F<\mathrm{AL}$ and $F>$ AL, being higher, $\alpha>a$, in the last one. This fact has an easy explanation; the no shortselling condition on the amount invested in the risky assets impose a higher rate of reduction on the contribution rate on the region $F>\mathrm{AL}$ than that would be in the control problem without constraints. If selling short is possible then the shape of $C(t)$ is

\footnotetext{
${ }^{4}$ However, it is an easy exercise to prove that the function appearing in (10) is a viscosity solution of (6). See Fleming and Soner (1992) for the appropriated definitions.
} 
the same in both regions. In this case, the reduction in the level of the fund when $F>\mathrm{AL}$ is fulfilled by following an investment strategy that takes high risk and low expected returns. With shortselling constraints, the contribution rate changes in order to getting back the fund to the ideal value. In this way the investment strategy in the region $F>$ AL is one of no risk and low expected returns. This result is in contrast with Boulier et al. (1995) and Cairns (2000). In the former paper, the stabilization of the funding process around a target is not a prior objective of the manager (note that the target would be increasing in time because the benefits are increasing too). On the other hand, the ideal contribution level is zero. These facts motivate the differences with our results. In the later paper different types of constraints are considered, in particular no borrowing and no shortselling. Although the model analyzed is quite similar to the one studied here, the coefficient of proportionality in the contribution rate is the same for all values of $F$. In our view, the problem becomes from the fact that in the presence of constraints the value function is no longer of quadratic type. This seems to be omitted in Cairns (2000). As is shown in Theorem 1, the shortselling constraint motivates that the value function is piecewise quadratic.

Let us note that borrowing at the risk-free rate of interest $r$ is optimal to be invested in asset $i\left(\lambda_{i}>1\right)$, whenever the fund level is below the critical value

$$
\frac{\mathbf{e}_{\mathbf{i}} \Sigma^{-1}(\mathbf{b}-r \mathbf{1})}{\mathbf{e}_{\mathbf{i}}\left(\mathbf{1}+\Sigma^{-1}(\mathbf{b}-r \mathbf{1})\right)} \mathrm{AL}
$$

where $\mathbf{e}_{\mathbf{i}}=\left(0, \ldots, 1^{i)}, 0, \ldots, 0\right)$.

The main concern of the promoter is to keep the contribution rate and the level of the fund as close as possible to the ideal values. As the following proposition shows, (15) and (16) implies the stabilization of the expected wealth of the fund on the desired target. In fact, an additional constraint on the weighing factor $\beta$ assure convergence a.s. of the fund to the actuarial liability.

Proposition 1. If (15) and (16) hold, then

1. The fund, the rate of contribution and the total investment converge a.s. as well as their conditional expectations to the actuarial liability, the normal cost and zero, respectively; that is to say,

$$
\lim _{t \rightarrow \infty} E_{F_{0}} F^{*}(t)=\mathrm{AL}, \quad \lim _{t \rightarrow \infty} E_{F_{0}} C^{*}(t)=\mathrm{NC} \quad \text { and } \quad \lim _{t \rightarrow \infty} E_{F_{0}} \Lambda^{*}(t) F^{*}(t)=0 .
$$

2. If the parameters of the problem verify

$$
a>\beta\left(r-\frac{1}{2} \theta^{\mathrm{T}} \theta\right),
$$

then the variance of the fund, the rate of contribution and the total investment amount converge to zero, that is to say,

$$
\lim _{t \rightarrow \infty} \operatorname{Var}_{F_{0}} F^{*}(t)=0, \quad \lim _{t \rightarrow \infty} \operatorname{Var}_{F_{0}} C^{*}(t)=0 \quad \text { and } \quad \lim _{t \rightarrow \infty} \operatorname{Var}_{F_{0}} \Lambda^{*}(t) F^{*}(t)=0 .
$$

Proof. See Appendix A.

The convergence properties of the fund to the actuarial liability can be strengthened as it is shown in the following proposition. We prove that the funding process never achieve the point AL with positive probability in finite time although this value is an attracting barrier. That is to say, the conditioned probability that $F^{*}$ takes the value $\mathrm{AL}$ before any other value tends to 1 as the gap between $\mathrm{AL}$ and $F^{*}$ shrinks.

Proposition 2. If (15) and (16) hold, AL is an unattainable and attracting barrier for $F^{*}$.

Proof. See Appendix A. 


\section{Conclusion}

This paper has analyzed the pension funding problem from the point of view of a manager that tries to minimize the solvency risk and the contribution rate risk on an unbounded horizon. To this end he or she makes a portfolio choice with $n$ risky assets and a bond and also controls the size of the contribution rate. We are able to give a closed form solution to the problem and we also prove strong stability properties of the solution. A spread method of funding arises, as a result of the postulated optimal behavior, that is to say, the supplementary cost is proportional to the unfunded actuarial liability. The shortselling constraints motivates the existence of two regions $(F<\mathrm{AL}$ and $F>\mathrm{AL}$ ) where the constant of proportionality is different. In the second region is higher than in the first one. This fact has important practical consequences. In order to guide the fund to the lower level AL, the manager has to diminish the contribution rate more than it is increased when the fund is below AL. At the same time, the total amount of the fund is invested in the risk-free bond. This is because the liabilities outgo are valued at this risk of return. When the fund is below the ideal value, the investment in high return, high risk assets decreases linearly as the fund increase to the ideal value. On the other hand, there exists a critical value of the fund such that it is optimal to borrow at the risk-free rate of interest to invest in the risky assets. As already noted by Boulier et al. (1996), Cairns (1996, 2000), this strategy is rather counterintuitive: it implies to take more and more risk when the level of the fund is low and it demands to put the fund in low risk assets when the fund is operating with large surplus.

In many countries funding legislation impose a minimum amount in cash for the fund to cover immediate benefit payments. Hence an interesting question is how affects the solution the presence of borrowing constraints. We suspect that in this case the supplementary cost is no longer proportional to the unfunded actuarial liability.

The hypothesis that the valuation force of interest equals the rate of return of the risk-free bond simplifies the algebra. If the equality does not holds, then the fund does not converges exactly to the actuarial liability. The same applies to the contribution rate and the normal cost.

In further research, we will propose to eliminate the constancy assumption on the actuarial (deterministic) functions used in the model. Suitable hypothesis from a tractable point of view would include exponential growth for the population of the plan and the salary. The autonomous character of the optimal control problem could be preserved in this case with the introduction of additional state variables (the normal cost, the actuarial liability and the benefits). We shall also consider modeling uncertainty on some elements of the fund. Again a tractable model would be the stochastic, exponential growth model.

\section{Appendix A}

In this appendix, we will give proofs of Propositions 1 and 2.

Proof of Proposition 1. By substituting the optimal values of the control variables, the evolution of the fund is given by the stochastic differential equation

$$
\mathrm{d} F^{*}(t)=\left(r F^{*}(t)+\theta^{\mathrm{T}} \theta \mathrm{UAL}^{*}(t)+\frac{a}{\beta} \mathrm{UAL}^{*}(t)+\mathrm{NC}-P\right) \mathrm{d} t+\mathrm{UAL}^{*}(t) \theta^{\mathrm{T}} \mathrm{d} \mathbf{W}(t)
$$

with $F^{*}(0)=F_{0}$, whenever $F^{*}<\mathrm{AL}$ and by the ordinary differential equation

$$
\mathrm{d} F^{*}(t)=\left(\frac{\alpha}{\beta}-r\right)\left(\mathrm{AL}-F^{*}(t)\right) \mathrm{d} t
$$

with $F^{*}(0)=F_{0}$ if $F^{*} \geq \mathrm{AL}$.

The first expression can be rewritten in terms of the unfunded actuarial liability as follows:

$$
\mathrm{dUAL}^{*}(t)=\left(r-\theta^{\mathrm{T}} \theta-\frac{a}{\beta}\right) \mathrm{UAL}^{*}(t) \mathrm{d} t-\mathrm{UAL}^{*}(t) \theta^{\mathrm{T}} \mathrm{d} \mathbf{W}(t)
$$


with $\mathrm{UAL}^{*}(0)=\mathrm{AL}-F_{0}$. The solution of this equation, a geometrical Brownian motion, is given by

$$
\mathrm{UAL}^{*}(t)=\left(\mathrm{AL}-F_{0}\right) \exp \left\{\left(r-\frac{3}{2} \theta^{\mathrm{T}} \theta-\frac{a}{\beta}\right) t-\theta^{\mathrm{T}} W(t)\right\} .
$$

Letting $t$ to infinity and taking into account (15) and the properties of Brownian motion, we have that UAL* converges to zero a.s. The conditional expected value of $\mathrm{UAL}^{*}$ is

$$
E_{F_{0}} \mathrm{UAL}^{*}(t)=\left(\mathrm{AL}-F_{0}\right) \exp \left\{\left(r-\theta^{\mathrm{T}} \theta-\frac{a}{\beta}\right) t\right\},
$$

that converges to zero by assumption (15). On the other hand (20) implies

$$
\lim _{t \rightarrow \infty} E_{F_{0}}\left(\mathrm{UAL}^{*}(t)\right)^{2}=0
$$

see (Arnold 1974, p. 140). Hence

$$
\operatorname{Var}_{F_{0}} \operatorname{UAL}^{*}(t)=E_{F_{0}}\left(\mathrm{UAL}^{*}(t)\right)^{2}-E_{F_{0}}^{2}\left(\mathrm{UAL}^{*}(t)\right)
$$

tends to zero as $t$ goes to infinity.

In the region $F^{*} \geq \mathrm{AL}$ the evolution of $\mathrm{UAL}^{*}$ is given by

$$
\mathrm{d} \mathrm{UAL}^{*}(t)=\left(r-\frac{\alpha}{\beta}\right) \mathrm{UAL}^{*}(t) \mathrm{d} t, \quad \mathrm{UAL}^{*}(0)=\mathrm{AL}-F_{0} .
$$

In this case it is clear that UAL* converges to zero when (16) holds. Now, the statements of the proposition are immediate consequences of (19), the properties of conditional expectations and variances and the behavior of UAL*.

Proof of Proposition 2. The statement of the proposition is equivalent to prove that 0 is an unattainable and attracting barrier for UAL* ${ }^{*}$ To this end, let us consider the natural scale function $\varphi$ of the diffusion process $\mathrm{UAL}^{*}$. It is defined as

$$
\varphi(x)=\int^{x} \exp \left(-\int^{y} \frac{2 \mu(z)}{a(z)} \mathrm{d} z\right) \mathrm{d} y,
$$

where

$$
\mu(x)=\left(r-\theta^{\mathrm{T}} \theta-\frac{a}{\beta}\right) x \quad \text { and } \quad a(x)=\theta^{\mathrm{T}} \theta x^{2}, \quad x>0
$$

are the drift and the diffusion coefficients of the process $\mathrm{UAL}^{*}$, respectively. We obtain

$$
\varphi(x)=(2 \gamma+1)^{-1} x^{2 \gamma+1}, \quad \text { where } \quad \gamma=1-\left(\theta^{\mathrm{T}} \theta\right)^{-1}\left(r-\frac{a}{\beta}\right)
$$

is a positive constant if (15) holds. The velocity density of the process is defined as

$$
m(x)=\left(a(x) \frac{\mathrm{d} \varphi}{\mathrm{d} x}(x)\right)^{-1}=\left(\theta^{\mathrm{T}} \theta\right)^{-1} x^{-2(\gamma+1)}, \quad x>0 .
$$

First we claim that 0 is unattainable for UAL*: if $x>0$

$$
\int_{0}^{x}(\varphi(y)-\varphi(0)) m(y) \mathrm{d} y=\left(\theta^{\mathrm{T}} \theta(2 \gamma+1)\right)^{-1} \int_{0}^{x} \frac{1}{y} \mathrm{~d} y=\infty
$$

and we can apply Karlin and Taylor (1981). If $x<0$, then the result follows from uniqueness of solutions to ordinary differential equations. Hence the claim follows. Second, we claim that 0 is attracting for UAL*. To show this, given 
$z>0$ let us consider $\tau_{z}^{*}$ the first instant of time such that UAL* hits $z$. The conditioned probability $P_{x}$ of the event $\tau_{z}^{*}>\tau_{0}^{*}$ when $\operatorname{UAL}(0)=x \in(0, z)$ is

$$
P_{x}\left(\tau_{z}^{*}>\tau_{0}^{*}\right)=\frac{\varphi(z)-\varphi(x)}{\varphi(z)-\varphi(0)}=\frac{z^{2 \gamma}-x^{2 \gamma}}{z^{2 \gamma}},
$$

see Karlin and Taylor (1981). It is clear that $P_{x}\left(\tau_{z}^{*}>\tau_{0}^{*}\right)$ as defined in (A.1) converges to 1 as $x$ goes to 0 .

\section{References}

Arnold, L., 1974. Stochastic Differential Equations: Theory and Applications. Wiley, New York.

Bellman, R., 1957. Dynamic Programming. Princeton University Press, Princeton, NJ.

Boulier, J.F., Trussant, E., Florens, D., 1995. A dynamic model for pension funds management. In: Proceedings of the Fifth AFIR International Colloquium, Vol. 1, pp. 361-384.

Boulier, J.F., Michel, S., Wisnia, V., 1996. Optimizing investment and contribution policies of a defined benefit pension fund. In: Proceedings of the Sixth AFIR International Colloquium, Vol. 1, pp. 593-607.

Bowers, N.L., Hickman, J.C., Nesbitt, C.J., 1976. Introduction to the dynamics of pension funding. Transactions of the Society of Actuaries 28 , 177-203.

Bowers, N.L., Hickman, J.C., Nesbitt, C.J., 1979. The dynamics of pension funding: contribution theory. Transactions of the Society of Actuaries 31, 93-119.

Cairns, A.J.G., 1995. Pension funding in a stochastic environment: the role of objectives in selecting an asset-allocation strategy. In: Proceedings of the Fifth AFIR International Colloquium, Vol. 1, pp. 429-453.

Cairns, A.J.G., 1996. Continuous-time stochastic pension fund modelling. In: Proceedings of the Sixth AFIR International Colloquium, Vol. 1, pp. 609-624.

Cairns, A.J.G., 2000. Some notes on the dynamics and optimal control of stochastic pension fund models in continuous time. Astin Bulletin $30,19-55$.

Daykin, C.D., Pentikäinen, T., Pesonen, M., 1994. Practical Risk Theory for Actuaries. Chapman \& Hall, London.

Fleming, W.H., Soner, H.M., 1992. Controlled Markov Processes and Viscosity Solutions. Springer, New York.

Haberman, S., 1993. Stochastic investment returns and the present value of future contributions in defined benefit pension schemes. Actuarial Research Paper, Vol. 49. City University.

Haberman, S., 1997. Stochastic investment returns and contribution rate risk in a defined benefit pension scheme. Insurance: Mathematics and Economics 19, 127-139.

Haberman, S., Sung, J.H., 1994. Dynamics approaches to pension funding. Insurance: Mathematics and Economics 15, 151-162.

Karatzas, I., Shreve, S.E., 1991. Brownian Motion and Stochastic Calculus, 2nd Edition. Springer, New York.

Karlin, S., Taylor, H.M., 1981. A Second Course on Stochastic Processes. Academic Press, New York.

Krylov, N.V, 1980. Controlled Diffusion Processes. Springer, New York.

Merton, R.C., 1971. Optimal consumption and portfolio rules in a continuous-time model. Journal of Economic Theory 3, $373-413$.

O'Brien, T.V., 1987. A two parameter family of pension contribution functions and stochastic optimization. Insurance: Mathematics and Economics 6, 129-134.

Owadally, M.I., Haberman, S., 1999. Pension fund dynamics and gains/losses due to random rates of investment return. North American Actuarial Journal 3, 105-117.

Vila, J.L., Zariphopoulou, T., 1997. Optimal consumption and portfolio choice with borrowing constraints. Journal of Economic Theory 77 , 402-431. 